\title{
Preparation and Detection of a Mechanical Resonator Near the Ground State of Motion
}

\author{
T. Rocheleau, ${ }^{1}$ T. Ndukum, ${ }^{1}$ C. Macklin, ${ }^{1}$ J.B. Hertzberg, ${ }^{2}$ A.A. Clerk,${ }^{3}$ and K.C. Schwab ${ }^{4,- \text { * }}$ \\ ${ }^{1}$ Department of Physics, Cornell University, Ithaca, NY 14853 USA \\ ${ }^{2}$ Department of Physics, University of Maryland, College Park, MD 20742 USA \\ ${ }^{3}$ Department of Physics, McGill University, Montreal, QC Canada H3A 2T8 \\ ${ }^{4}$ Applied Physics, Caltech, Pasadena, CA 91125 USA
}

(Dated: July 19, 2009)

\begin{abstract}
We have cooled the motion of a radio-frequency nanomechanical resonator by parametric coupling to a driven microwave frequency superconducting resonator. Starting from a thermal occupation of 480 quanta, we have observed occupation factors as low as $3.8 \pm 1.2$ and expect the mechanical resonator to be found with probability 0.21 in the quantum ground state of motion. Cooling is limited by random excitation of the microwave resonator and heating of the dissipative mechanical bath.
\end{abstract}

PACS numbers: 85.85.+j, 42.50.Wk, 84.40.Dc, 85.25.-j

Cold macroscopic mechanical systems are expected to behave contrary to our usual classical understanding of reality; the most striking and nonsensical predictions are states where the mechanical system is located in two places simultaneously. Various schemes have been proposed to generate and detect such states [1, 2] and all require starting from mechanical states which are close to the lowest energy eigenstate, the mechanical ground state.

Naively treating the motion of a mechanical resonator quantum mechanically, one finds the elementary result that the energy should be quantized: $E_{n}=\hbar \omega_{m}\left(n+\frac{1}{2}\right)$, where $n$ is an integer and $\omega_{m}$ is the resonant frequency. In thermal equilibrium, an average occupation factor is expected to follow the Bose-Einstein distribution: $\bar{n}_{m}^{T}=$ $\left(e^{\hbar \omega_{m} / k_{B} T}-1\right)^{-1}$, where $T, 2 \pi \hbar$, and $k_{B}$ are the temperature, Planck's and Boltzmann's constants respectively. Cooling a resonator into the quantum regime where $\bar{n}_{m}^{T}<<1$, and measuring the very small motions has been challenging for a number of technical reasons; not only are very low temperatures necessary to freezeout the mode, but detection with sensitivity at the quantum zero-point level is required: $x_{z p}=\sqrt{\hbar /\left(2 m \omega_{m}\right)}$, where $m$ is the resonator mass. Furthermore, this strong position measurement must not heat the mode with measurement backaction [3].

Many strategies have been proposed[4, 5, 6, 7, 8, 9, 10, 11, 12] and applied to realize the quantum regime with increasing success. Experiments with nanoelectromechanical structures have been able to reach $\bar{n}_{m}=25$ by passively cooling a nanomechanical resonator (NR) 3], detected with a superconducting single electron transistor [13]. Researchers experimenting with opto-mechanical systems have been able to utilize ultrasensitive optical detection and radiation pressure to both cool and detect $\bar{n}_{m}=65$ in a toroidal resonator [14, $\bar{n}_{m}=37$ in microsphere resonator [15], and $\bar{n}_{m}=35$ in an optical cavity [16].

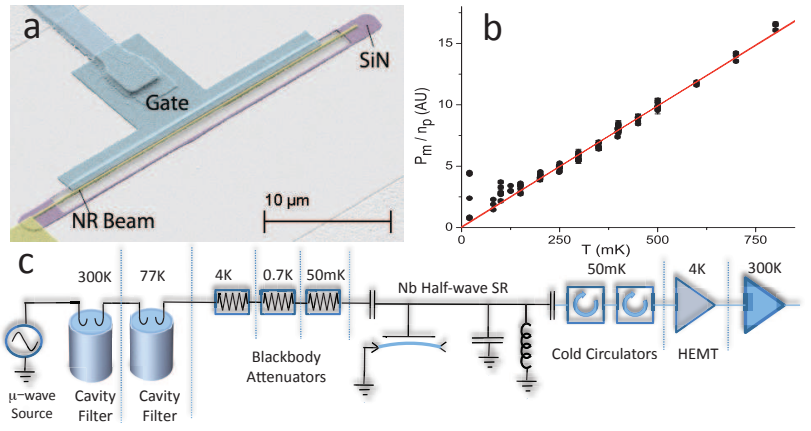

FIG. 1: a) Shows the $\mathrm{Nb} / \mathrm{Al} / \mathrm{SiN}$ sample: the $\mathrm{NR}$ is $30 \mu \mathrm{m}$ long, $170 \mathrm{~nm}$ wide, $140 \mathrm{~nm}$ thick, formed of $60 \mathrm{~nm}$ of stoichiometric, high-stress, LPCVD SiN 17 and 80nm of Al, and located $75 \mathrm{~nm}$ from the gate electrode connected to the SR. The SR is fabricated from a $345 \mathrm{~nm}$ thick $\mathrm{Nb}$ film and has a characteristic impedance of $126 \Omega$. (b) Shows the thermal calibration of the upconverted noise power. (c) Shows the ultra-low noise, cyrogenic measurement circuit.

The technique we employ both to cool and detect the motion of a NR close to the ground state involves parametrically coupling the motion to a superconducting microwave resonator (SR) [18, 19], (Fig. 1.) The NR has a fundamental in-plane flexural resonance of $\omega_{m}=2 \pi \cdot 6.3 \mathrm{MHz}$ and is capacitively coupled to a symmetric, two-port, half-wave SR which resonates at $\omega_{s r}=2 \pi \cdot 7.5 \mathrm{GHz}$. The device is located in a dilution refrigerator and pumped through carefully filtered and cooled leads. The thermal occupation of the $\mathrm{SR}, \bar{n}_{s r}^{T}$ is expected to be 0.09 at $146 \mathrm{mK}$.

The NR damping rate, $\Gamma_{m}^{T}$, displays an unusual linear temperature dependence below $600 \mathrm{mK}$, reaching $Q \sim$ $10^{6}$ at $100 \mathrm{mK}$. The SR damping rate, $\Gamma_{s r}=2 \pi \cdot 600 \mathrm{kHz}$, is essentially temperature independent below $700 \mathrm{mK}$ and is a factor of 2.4 higher than expected from design due to internal losses.

The Hamiltonian which describes the coupled res- 
onators is given by $[\underline{9},[10]$ :

$$
\hat{H}=\hbar\left(\omega_{s r}+g \hat{x}-\lambda \hat{x}^{2}\right)\left(\hat{b}^{\dagger} \hat{b}+\frac{1}{2}\right)+\hbar \omega_{m}\left(\hat{a}^{\dagger} \hat{a}+\frac{1}{2}\right)
$$

where $\hat{a}\left(\hat{a}^{\dagger}\right)$ and $\hat{b}\left(\hat{b}^{\dagger}\right)$ are the NR and SR annihilation (creation) operators. The first term shows the pondermotive-like coupling of the SR's frequency to the mechanical motion: $\hat{x}=x_{z p}\left(\hat{a}^{\dagger}+\hat{a}\right)$ and $g=\frac{\partial \omega_{s r}}{\partial x}=$ $\frac{\omega_{s r}}{2 C_{t}} \frac{\partial C_{g}}{\partial x}$ where $C_{g}(x)=450 \pm 50 a F$ is the coupling capacitance and $C_{t}=260 \mathrm{fF}$ is the SR total effective capacitance. The term proportional to $\hat{x}^{2}$ results from the electrostatic frequency pulling of the mechanical resonator by the SR[20], where $\lambda=\frac{\omega_{s r}}{2 C_{t}} \frac{\partial^{2} C_{g}}{\partial x^{2}}$, and is responsible for parametric instabilities under certain pump configurations 21].

When pumping the SR at $\omega_{p}=\omega_{s r}-\omega_{m}$, harmonic motion of the NR preferentially up-converts microwave photons to frequency $\omega_{s r}$, extracting one radiofrequency NR quantum for each up-converted microwave SR photon, a process which both damps and cools the NR motion [9, 10, 11, 22, 23]. This cooling process is analogous to Raman scattering and the process used to cool an atomic ion to the quantum ground state of motion [10, 24]. In the sideband-resolved limit, $\Gamma_{s r}<$ $\omega_{m}$, the rate of this up-conversion process is given by: $\Gamma_{\text {opt }}=4 x_{z p}^{2} g^{2} \bar{n}_{p} / \Gamma_{s r}$, where $\bar{n}_{p}$ is the occupation of the SR from the pumping.

From detailed balance, the NR occupation factor is expected to follow:

$$
\bar{n}_{m}=\frac{\Gamma_{m}^{T} \bar{n}_{m}^{T}+\Gamma_{o p t} \bar{n}_{s r}}{\Gamma_{m}^{T}+\Gamma_{o p t}}
$$

where $\bar{n}_{s r}=\left(\Gamma_{s r} /\left(4 \omega_{m}\right)\right)^{2}+\bar{n}_{s r}^{T}\left[1+2\left(\Gamma_{s r} /\left(4 \omega_{m}\right)\right)^{2}\right]$ is the effective occupation factor of the SR when $\Gamma_{o p t}<\Gamma_{s r}[25]$. The first term in the expression for $\bar{n}_{s r}$ is due to the quantum fluctuations of the pump field, and the second term due to the thermal occupation of the $\mathrm{SR}, \bar{n}_{S R}^{T}$. The expressions above show that the minimum mechanical occupation possible is the effective occupation of the SR.

The first realization of cooling in a parametrically coupled, electro-mechanical microwave system was with a kg-scale gravitational wave transducer [26], cooling from $\bar{n}_{m}=10^{8}$ to $10^{5}$; cooling of an NR with an SR was recently demonstrated and achieved cooling from $\bar{n}_{m}=700$ to 120 27.

The up-converted noise power is calibrated by applying a weak pump signal, $\left(\Gamma_{o p t}<\Gamma_{m}^{T}\right)$, and measuring the resulting integrated sideband power, $P_{m}$, versus refrigerator temperature, T (Fig. 1b.) For temperature above $\sim 150 \mathrm{mK}$, we observe the expected behavior consistent with Equipartition and use this curve to establish the relationship between measured output noise power and $\bar{n}_{m}$. For temperatures below $150 \mathrm{mK}$ we observe fluctuations in $\bar{n}_{m}$ apparently due to a non-thermal, intermittent, force noise at the level of $1 \cdot 10^{-18} \mathrm{~N} / \sqrt{\mathrm{Hz}}$ which

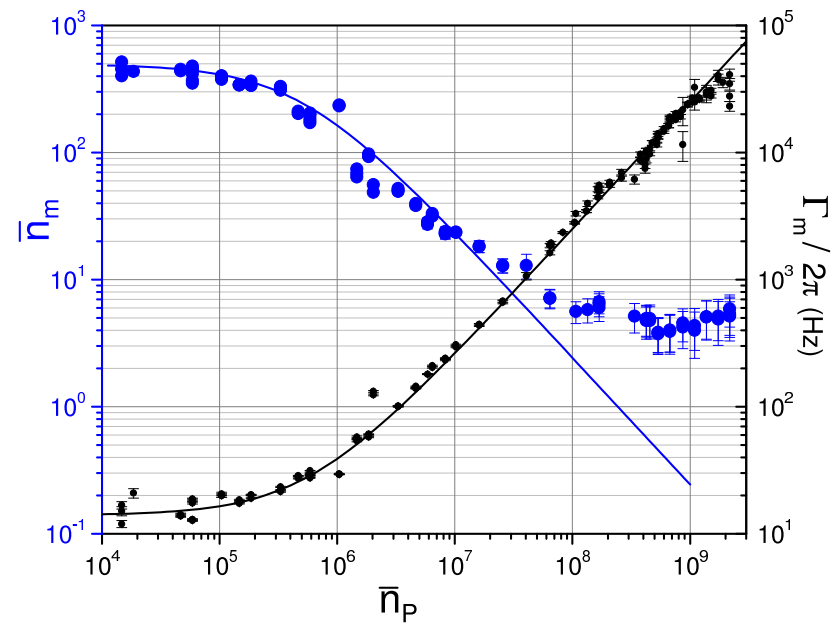

FIG. 2: The figure shows $\bar{n}_{m}(\bullet)$ and $\Gamma_{m}=\Gamma_{m}^{T}+\Gamma_{o p t}(\bullet)$ versus $\bar{n}_{p}$. The solid black curve is a fit to the measured $\Gamma_{m}$. The solid blue curve is the expected value of $\bar{n}_{m}$ assuming ideal values of $\bar{n}_{S R}$ and $\dot{n}_{T}=3 \cdot 10^{4}$ quanta/sec.

is observed in other similar samples 21, 28] and similar to anomalous heating effects in other systems [29, 30]. Furthermore, the linear temperature dependence of $\Gamma_{m}^{T}$ causes the NR to decouple from the thermal environment at the lowest measured temperatures.

The measured signal powers are consistent with our knowledge of the attenuation and gain of our measurement circuit, and estimates of the device parameters. We find $g / 2 \pi=84 \pm 5 \mathrm{kHz} / \mathrm{nm}$, which is the largest coupling strength demonstrated to date in a system of this type. From measurements of $\omega_{m}$ versus $\bar{n}_{p}$ and pump frequency, we determine $\lambda / 2 \pi=2.1 \pm 0.7 \mathrm{kHz} /(\mathrm{nm})^{2}$.

Figure 2 shows the central result of this work. With the refrigerator stabilized at $\mathrm{T}=146 \mathrm{mK}\left(\bar{n}_{m}^{T}=480\right)$ we measure $\Gamma_{m}$ and $\bar{n}_{m}$ versus the SR pump occupation, $\bar{n}_{p}$. As is clear from Fig. 2, this process dramatically cools the motion. However, we also observe that the SR becomes increasingly excited as $\bar{n}_{p}$ is increased(Fig. 4).

Figure 3 shows the measured output noise spectra, $S_{x}(\omega)$, which is composed of up-converted microwave photons due to $\bar{n}_{m}$, SR noise due to $\bar{n}_{s r}$, and HEMT amplifier noise. Correlations between the NR motion and the SR field are important in our measured noise spectra at the lowest mechanical occupation factors. Fluctuations in the SR voltage, due to $\bar{n}_{s r}$, together with the pump, produce forces at the $\omega_{m}$. The resulting motion, together with the pump, produces noise at $\omega_{s r}$, however $180^{\circ}$ out of phase with the original SR fluctuations. This correlation results in an inverted noise peak 21], similar to noise squashing 31], which adds incoherently to the noise power driven by the thermal bath. Our analysis shows that the NR occupation factor is given by $\bar{n}_{m}=\bar{n}_{e f f}+2 \bar{n}_{s r}$, where $\bar{n}_{\text {eff }}$ is the occupation measured directly from the integrated noise peak (or dip) in 


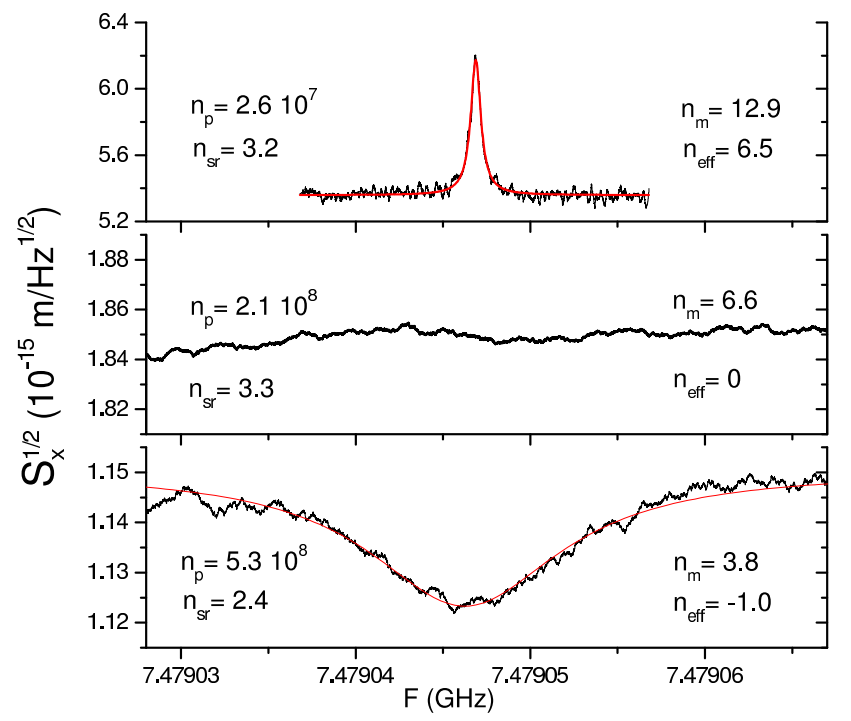

FIG. 3: shows the noise squashing effect on $S_{x}(\omega)$ due to the finite occupation of the SR, in three situations: when $\bar{n}_{\text {eff }}>0$ (top), when $\bar{n}_{\text {eff }} \approx 0$ (middle), and when $\bar{n}_{\text {eff }}<0$ (bottom). The red curves show Lorentzian fits through the mechanically up-converted side-band.

the output noise spectrum, $S_{x}(\omega)$. Figure 3 shows measurements of $S_{x}(\omega)$ in three cases at low occupation factors: when $\bar{n}_{\text {eff }}>0$, when $\bar{n}_{\text {eff }} \approx 0$, and when $\bar{n}_{e f f}<0$ showing the squashed output noise.

Taking the effects of $\bar{n}_{s r}$ into account in this way, the lowest mechanical occupation we have observed is $\bar{n}_{m}=$ $3.8 \pm 1.2$, shown in Fig. 3, with the uncertainty dominated by the uncertainty in $\bar{n}_{s r}$. At this low occupation factor the resonator is expected to be found in the ground state with probability $P_{0}=1 /\left(\bar{n}_{m}+1\right)=0.21$. The cooling power of this refrigeration technique is $\dot{Q}=\hbar \omega_{m} \cdot \Gamma_{\text {opt }}=$ $10^{-22} \mathrm{~W}$

We have lowered the refrigerator temperature to $20 \mathrm{mK}$ and do not observe an decrease in the minimum $\bar{n}_{m}$. Using the detailed balance relationship and the measured $\bar{n}_{m}$ and $\Gamma_{s r}$, we can compute the bath heating rate, $\dot{n}_{T}=\Gamma_{m}^{T} \bar{n}_{m}^{T}$, versus $\bar{n}_{p}$, (Fig. 4.) It is clear that as $\bar{n}_{p}$ increases above $3 \cdot 10^{7}, \dot{n}_{T}$ begins to increase, nullifying the benefit of starting at low temperatures. This level of heating is consistent with ohmic losses in the metal film on top the NR, and the thermal conductance of a normal-state electron gas.

Current Limitations and Future Directions: These measurements identify three effects which work against the cooling process: excess fluctuations of the $\mathrm{SR}\left(\bar{n}_{S R}\right)$, heating of the NR thermal bath at high pump powers, and the non-thermal force noise at low temperatures.

We believe that the excess SR occupation, $\bar{n}_{s r}$, is not a result of phase or amplitude noise of our microwave source: the pump signal is filtered with tunable, copper microwave cavities (one at $300 \mathrm{~K}\left(\mathrm{Q}=9.5 \cdot 10^{3}\right)$ followed by a second at $\left.77 \mathrm{~K}\left(\mathrm{Q}=2.6 \cdot 10^{4}\right)\right)$ achieving bet-

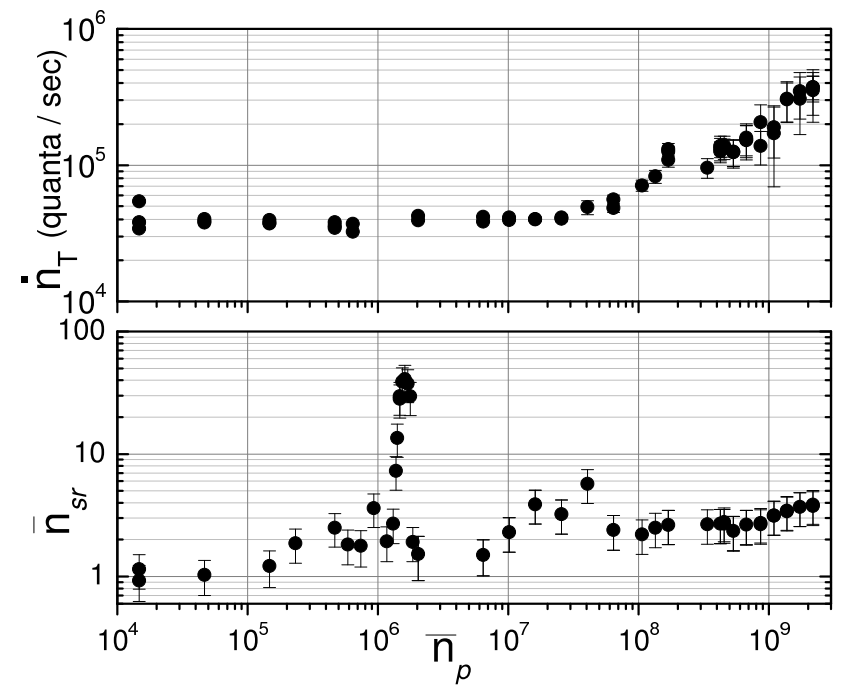

FIG. 4: The upper figure shows the bath heating rate, $\dot{n}_{T}$, versus pump strength $\bar{n}_{p}$, and the onset of excess heating above $\bar{n}_{p}=3 \cdot 10^{7}$. The lower figure shows the measured value of $\bar{n}_{s r}$ versus $\bar{n}_{p}$; the structure is suspected to be related to temporal dynamics of the transition between superconducting and normal states of the metal films and resulting microwave side-band generation 32].

ter than $\mathcal{L}(+6.3 M H z)<-195 \mathrm{db}_{c} / \mathrm{Hz}$, and contributing less than 0.04 photons into the $\mathrm{SR}$ at our highest value of $\bar{n}_{p}$. Without these cavities the $\mathrm{SR}$ would be excited to $\bar{n}_{s r}=35$. We also believe that this excess SR occupation is not due to ohmic heating of and resulting thermal radiation from the cyrogenic attenuator network since $\bar{n}_{s r}$ increases only weakly over a wide span of $\bar{n}_{p}$. Tests of Nb $\mathrm{SR}$ devices at $1.2 \mathrm{~K}$ before the surface micromaching of the NR do not show excess dissipation and suggest that the excess losses are related to our fabrication process.

Increasing $\Gamma_{\text {opt }}$ by engineering larger coupling strength, $g$, and/or decreasing $\Gamma_{s r}$ should be very beneficial since it will lead to higher cooling rates at lower pump powers, minimizing the effect of excess bath heating, $\dot{n}_{T}$. By increasing $\Gamma_{\text {opt }}$ a factor of 10 , maintaining the same $\dot{n}_{T}$, we expect $\bar{n}_{m} \approx 0.5$, with $P_{0}=.67$. This approach will be limited when $\Gamma_{\text {opt }}$ becomes comparable to $\Gamma_{s r}$ which limits the rate of cooling 25, 33].

The deep quantum limit, $\bar{n}_{m} \ll 1$, will be accessible when it is possible to utilize lower refrigerator temperatures and lower mechanical damping rates at these temperatures. Understanding and eliminating the excess bath heating and the non-thermal force noise will be required. Furthermore, superconducting metals on the NR also appear to be required due to the expected mechanical force noise from transport and electron momentum scattering in diffusive conductors [34, 35]. We estimate that this heating mechanism will limit $\bar{n}_{m}>3$ at $\bar{n}_{p}=3 \cdot 10^{8}$ assuming our current device parameters and a resistance of $100 \Omega$ through the NR. 
These measurements show that detection with sensitivity to resolve motions approaching the ground state is possible with existing HEMT-based amplifiers. Eliminating internal SR losses, unbalancing the SR couplings, and implementing improved microwave amplifiers [36, 37] would significantly reduce the measurement time.

Nonetheless, the production and detection of a NR with $\bar{n}_{m}=3.8$ is sufficient to enable future experiments. Due to Uncertainy Principle fluctuations of the mechanical motion and resulting spontaneous emission, the rate of microwave photon up-conversion is expected to differ from the rate of down-conversion. This difference can be used as a fundamental thermometry technique 9, 10, 24, and would be the first quantitative measurement of the zero-point motion of a mechanical structure.

This level of cooling is essential to realize entangled states between superconducting quantum bits and the motion of a nanomechanical device [1, 38, 39]. Similar to procedures in atomic physics, such an experiment would involve preparing the cold state of the mechanical device and after the refrigeration is complete, the cooling can be turned off. The state of the cold beam could then be manipulated before thermallization of the motion. In our realization, we expect cooling from $\bar{n}_{m}=500$ to 4 quanta in $\sim 200 \mu \mathrm{s}$, and one thermal quantum to enter the resonator in $\tau=\left(\dot{n}_{T}\right)^{-1}=2 \mu$ s which exceeds superconducting qubit manipulation times and is comparable to qubit measurement and relaxation times.

We acknowledge helpful conversations with M. Aspelmeyer, R. Ilic, M. Skvarla, M. Metzler, M. Shaw and assistance from M. Savva, S. Rosenthal, and M. Corbett. This work has been supported by the Fundamental Questions Institute fqxi.org (RFP2-08-27), and the US NSF (DMR-0804567). Device fabrication was performed at the Cornell Nanoscale Facility, a member of the NNIN (NSF Grant ECS-0335765).

* schwab@caltech.edu www.kschwabresearch.com

[1] A. Armour, M. Blencowe, and K. Schwab, Phys. Rev. Lett. 88, 148301 (2002).

[2] W. Marshall, C. Simon, R. Penrose, and D. Bouwmeester, Phys. Rev. Lett. 91, 130401 (2003).

[3] A. Naik, O. Buu, M. D. LaHaye, A. D. Armour, A. A. Clerk, M. D. Blencowe, and K. C. Schwab, Nature 443 , 193 (2006).

[4] J. M. Courty, A. Heidmann, and M. Pinard, Eur. Phys. J. D 17, 399 (2001).

[5] A. Hopkins, K. Jacobs, S. Habib, and K. Schwab, Phys. Rev. B 68, 235328 (2003).

[6] I. Wilson-Rae, P. Zoller, and A. Imamoglu, Phys. Rev. Lett. 92, 075507 (2004).

[7] I. Martin, A. Shnirman, L. Tian, and P. Zoller, Phys. Rev. B 69, 125339 (2004).

[8] M. P. Blencowe and E. Buks, Phys. Rev. B 76, 014511 (pages 16) (2007).
[9] F. Marquardt, J. P. Chen, A. A. Clerk, and S. M. Girvin, Phys. Rev. Lett. 99, 093902 (2007).

[10] I. Wilson-Rae, N. Nooshi, W. Zwerger, and T. J. Kippenberg, Phys. Rev. Lett. 99, 093901 (2007).

[11] F. Xue, Y. D. Wang, Y. Liu, and F. Nori, Phys. Rev. B 76, 205302 (2007).

[12] L. Tian, Phys. Rev. B 79, 193407 (2009).

13] M. D. LaHaye, O. Buu, B. Camarota, and K. C. Schwab, Science 304, 74 (2004).

[14] Y.-S. Park and H. Wang, Nature Phys. (2009).

[15] A. Schliesser, O. Arcizet, R. Riviere, G. Anetsberger, and T. J. Kippenberg, Nature Phys. (2009).

[16] X. M. H. Huang, C. A. Zorman, M. Mehregany, and M. L. Roukes, Nature Phys. (2009).

[17] S. S. Verbridge, H. G. Craighead, and J. M. Parpia, Appl. Phys. Lett. 92, 013112 (2008).

[18] P. K. Day, H. G. LeDuc, B. A. Mazin, A. Vayonaksi, and J. Zmuidzinas, Nature 425, 817 (2003).

[19] C. A. Regal, J. D. Teufel, and L. W. Lehnert, Nature Phys. 4, 555 (2008).

[20] A. N. Cleland and M. L. Roukes, Nature 392, 160 (1998).

[21] J. B. Hertzberg, T. Rocheleau, T. Ndukum, M. Savva, A. A. Clerk, and K. C. Schwab, arXiv:0906.0967 (2009).

[22] M. I. Dykman, Sov. Phys. Solid State 20, 1306 (1978).

[23] N. P. Linthorne, P. J. Veitch, and D. G. Blair, J. Phys. D: Appl. Phys. 23, 1 (1990).

[24] F. Diedrich, J. Bergquist, W. Itano, and D. Wineland, Phys. Rev. Lett. 62, 403 (1989).

[25] J. M. Dorbrindt, I. Wilson-Rae, and T. J. Kippenberg, Phys. Rev. Lett. 101, 263602 (2008).

[26] D. G. Blair, E. N. Ivanov, M. E. Tobar, P. J. Turner, F. van Kann, and I. S. Heng, Phys. Rev. Lett. 74, 1908 (1995).

[27] J. D. Teufel, J. W. Harlow, C. A. Regal, and K. W. Lehnert, Phys. Rev. Lett. 101, 197203 (2008).

[28] J. D. Teufel, C. A. Regal, and K. W. Lehnert, New J. Phys. 10, 095002 (2008).

[29] B. C. Stipe, H. J. Mamin, T. D. Stowe, T. W. Kenny, and D. Rugar, Phys. Rev. Lett. 87, 096801 (2001).

[30] L. Deslauriers, S. Olmschenk, D. Stick, W. K. Hensinger, J. Sterk, and C. Monroe, Phys. Rev. Lett. 97, 103007 (2006).

[31] M. Poggio, C. L. Degen, H. J. Mamin, and D. Rugar, Phys. Rev. Lett. 99, 017201 (2007).

[32] E. Arbel-Segev, B. Abdo, O. Shtempluck, and E. Buks, Condens. Matter 19, 096206 (2007).

[33] M. Grajcar, S. Ashhab, J. R. Johansson, and F. Nori, Phys. Rev. B 78, 035406 (2008).

[34] A. V. Shytov, L. S. Levitov, and C. W. J. Beenakker, Phys. Rev. Lett. 88, 228303 (2002).

[35] P. A. Truitt, Ph.D. thesis, Univ. of Maryland, College Park, MD (2007).

[36] M. Mück, C. Welzel, and J. Clarke, Applied Physics Letters $\mathbf{8 2}, 3266$ (2003).

[37] M. A. Castellanos-Beltran, K. D. Irwin, G. C. Hilton, L. R. Vale, and K. W. Lehnert, Nature Phys. 4, 929 (2008).

[38] D. W. Utami and A. A. Clerk, Phys. Rev. A 78, 042323 (2008).

[39] M. D. LaHaye, J. Suh, P. M. Echternach, K. C. Schwab, and M. L. Roukes, Nature 459, 960 (2009). 